\title{
Differences in knee skin temperature between left and right leg after running on a treadmill measured by infrared thermography
}

\author{
by V. Svaic* and I. Jurak*
}

\author{
* University of Applied Health Sciences, Mlinarska cesta 38, Zagreb, Croatia, vjeran.svaic@skole.hr
}

\begin{abstract}
The main goal of this study was to analyse temperature differences between left and right knees after the graded exercise by running on a treadmill in closed environment. Nine young athletes conducted a submaximal test and thermography was used to determine quantitative measurement of knee skin temperature before and immediately after the exercise. Significant difference was found between left and right leg after the exercise, suggesting that both legs are not used the same way with one leg doing more work, thus showing different temperatures.
\end{abstract}

\section{Introduction}

During the last thirty years thermography found its use in sport and exercise science where studies are usually based on skin temperature changes in different climate conditions or exercise types [1]. The thermo-regulatory system of the human body maintains a constant temperature against a wide range of environmental and/or physical work conditions [2]. It is well known that physical activity induces a complex thermoregulation process where part of heat is given off by the skin of athlete. Skin temperature is determined by multiple factors such as a combination of conduction of the heat from the working musculature, perfusion of the blood and heat exchange between human body and the environment. Physical activity increases central body temperature due to the higher production of energy in involved musculature during physical work [3]. Previous studies have found a decrease in temperature on the surface of the skin during physical activity and an increase in temperature during the recovery phase [3,4]. Different studies have proved hypothesis that temperatures of left and right sides of the body are symmetrical [5]. On the other hand Hildebrandt et al. [6] address the temperature differences of $0,7^{\circ} \mathrm{C}$ or higher can be considered abnormal and potentially indicate physiological or anatomical differences in locomotor system.

Heart rate monitoring and IRT can give us useful non-invasive information of physiological activity of an athlete body, especially when taken into consideration latest studies which show that anaerobic effort is accompanied by a substantial drop of the temperature on surface of engaged muscles and the degree of the drop is proportional to the blood lactate concentration [7]. Secondly, skin temperature can be used as a physiological indicator of energy expenditure [8]. Simple methods for acquisition of general anaerobic threshold indicators exist (some basic instruments are necessary) and are based on the heart rate and load intensity relations analysis. Namely, heart rate is linearly correlated with lower loads intensity [9]. Both laboratory and field tests are used to acquire basic indicators of athletes aerobic capacity.

In this study we used adapted graded treadmill exercise test protocol to determine differences in skin temperatures between left and right leg over the specific region of interest over the knee joint. The test was carried out in a laboratory conditions (closed exercise room). This test is an indicator of the aerobic capacity and is used mostly in athletes for initial, transit or final measurements during the training process. The higher the aerobic capacity, the better conditioning an athlete has.

\section{Methods}

Nine amateur athletes, 18 years of age, conducted graded aerobic test by running on a treadmill. The test was adapted for the needs of this study with monitoring of the heart rate and running speeds, but excluding the measurements of oxygen consumption. Subjects were given instructions that they should not consume caffeine or smoke cigarettes in the morning prior to the testing. They were also asked not to participate in any training prior to the testing. All the subjects declared they had followed the instructions. Upon the arrival to the exercise room, they changed to their sport shorts and t-shirt. Shorts were kept above the knees region of interest (ROI) so they would not be touching the skin during running or imaging. Thermography measurements were made following the Glamorgan protocol procedure and using the proposed regions of interest (ROI) of the knee (fig. 1), with no direct air flow or light being emitted near the measuring area. The subjects acclimatised in the room temperature and humidity for 15 minutes prior to the testing. Height, weight and body mass index (BMI) was measured prior to the acclimatisation period, while initial knee temperature was measured after the acclimatisation period. The subjects were then ready to, one by one, take part in the testing procedure. Starting speed of the treadmill was set to $3.5 \mathrm{~km} / \mathrm{h}$ with $5 \%$ incline. Speed was increased by $0.5 \mathrm{~km} / \mathrm{h}$ every minute. The test was conducted until the maximum running intensity was achieved. This intensity was individual so the time and final running speed we different for every subject. Final thermogram was acquired immediately after the 
running was finished, within the first 30 seconds. This time of the recovery phase in which thermography imaging is conducted is crucial in interpreting the results and should be specified as a variable in future research. Mean temperature in the training room was $25.8( \pm 1.5){ }^{\circ} \mathrm{C}$ and relative humidity $29.9( \pm 4.6) \%$. Descriptive statistics of the athletes can be seen in table 1. Average heart rate $(\mathrm{HR})$ of the subjects during the resting period before running was $72.4( \pm 12.0)$.

The data was analysed using repeated measures ANOVA and post-hoc t-tests with Bonferroni correction for multiple testing. Pearson's correlation was calculated to ascertain association of BMl and resting knee temperatures. Statistical analysis was performed using statistical software R (ver. 3.6.2.). Following R packages were used in the analysis: rcompanion, expss, readxl, xlsx, foreign, car, corrplot, ggcorrplot, data.table, table1, effects, nlme, multcomp, knitr, tidyverse, ggpubr.

Table 1. Sample descriptives

\begin{tabular}{|l|c|c|c|c|}
\hline \multicolumn{1}{|c|}{ Variable } & Range & Minimum & Maximum & Mean [SD] \\
\hline Age (years) & 0.0 & 18.0 & 18.0 & $18.0[0.0]$ \\
\hline Height $(\mathrm{cm})$ & 20.0 & 168.0 & 188.0 & $180.4[7.2]$ \\
\hline Weight $(\mathrm{kg})$ & 44.0 & 58.0 & 102.0 & $77.1[16.2]$ \\
\hline BMI & 12.0 & 18.8 & 30.8 & $23.6[4.0]$ \\
\hline HR - rest & 39.0 & 48.0 & 87.0 & $72.4[12.0]$ \\
\hline HR - maximum & 36.0 & 168.0 & 204.0 & $191.1[10.9]$ \\
\hline
\end{tabular}

${ }^{\star} \mathrm{BMI}$ - body mass index, SD - standard deviation, HR - heart rate

\section{Results}

Average start of running was at $7.17( \pm 0.41) \mathrm{km} / \mathrm{h}$ and total running time of subjects was $13.1( \pm 1.97)$ minutes. Subjects achieved maximum HR during treadmill run of $191.1( \pm 10.8)$ beats per minute. Right and left knees skin temperature was measured from the anterior side during resting period with average values $29.89( \pm 1.19){ }^{\circ} \mathrm{C}$ and 29.76 $( \pm 1.01)^{\circ} \mathrm{C}$ respectively. Skin over the right knee was cooler after the exercise by $2.79{ }^{\circ} \mathrm{C}$ and left knee by $2.83{ }^{\circ} \mathrm{C}$. Results of the measured descriptive variables are shown in table 2.

Table 2. Descriptive statistics of measured variables

\begin{tabular}{|l|c|c|}
\hline Variable & Mean (SD) & Median [Min, Max] \\
\hline Right knee - rest & $29.89(1.19)$ & $30.30[27.51,31.51]$ \\
\hline Left knee - rest & $29.76(1.01)$ & $30.13[27.71,31.23]$ \\
\hline Right knee - activity & $27.10(1.49)$ & $27.81[25.27,29.40]$ \\
\hline Left knee - activity & $26.93(1.37)$ & $27.33[25.30,29.18]$ \\
\hline Test duration & $13.11(1.97)$ & $13.00[10.00,17.00]$ \\
\hline Speed at the start of run & $7.17(0.41)$ & $7.00[6.50,8.00]$ \\
\hline
\end{tabular}

Repeated measures ANOVA showed no significant interaction effect. Both main effects, difference of the knee temperatures before and after the exercise, as well as difference of the mean temperature between legs, were both significant $(F(1,8)=47.59 ; p<0.001$ and $F(1,8)=6.08 ; p=0.04)$, respectively). Partial eta squared $\left(\eta^{2} p\right)$ shows large effect size for before and after the exercise and medium effect size for difference between legs.

Table 3. Mixed model ANOVA

\begin{tabular}{|l|c|c|c|c|c|c|c|c|c|}
\hline Variable & \multicolumn{3}{|c|}{ Before - after (BA) } & \multicolumn{3}{c|}{ Right - left (RL) } & \multicolumn{3}{c|}{ BA x RL (interaction) } \\
\hline & $\mathbf{F}$ & $\boldsymbol{p}$ & $\mathbf{n}_{\mathbf{p}}^{2}$ & $\mathbf{F}$ & $\boldsymbol{p}$ & $\mathbf{\eta}_{\mathbf{p}}^{2}$ & $\mathbf{F}$ & $\boldsymbol{p}$ & $\mathbf{\eta}_{\mathrm{p}}{ }^{2}$ \\
\hline Average knee temp. & 47,59 & $<.001^{\star}$ & .86 & 6.08 & $=.04$ & .43 & 0.54 & $>.05$ & .06 \\
\hline
\end{tabular}




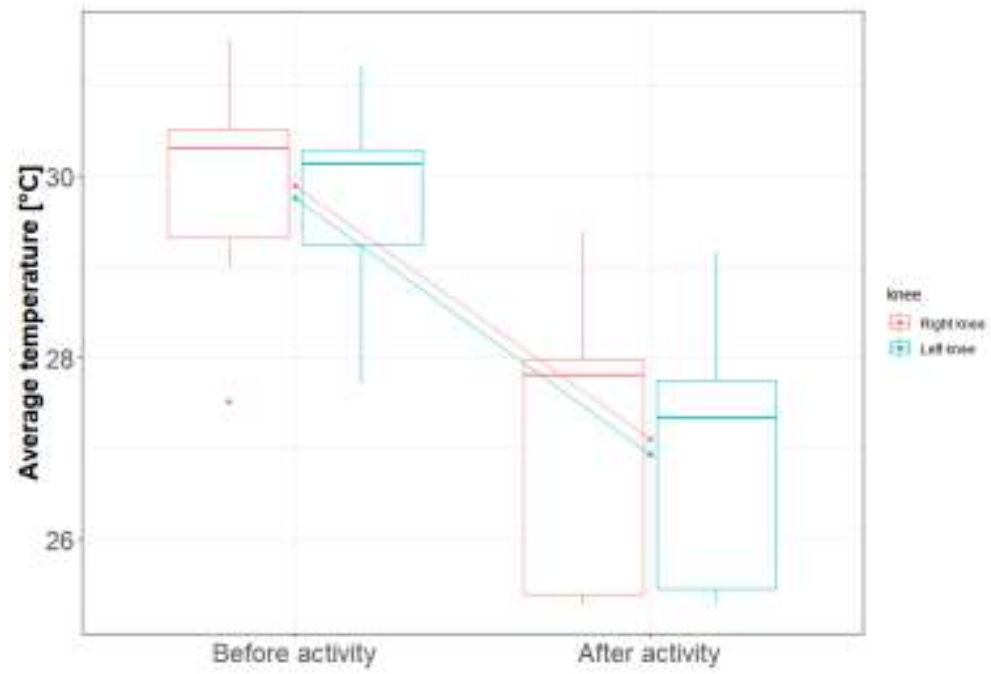

Plot 1. Interaction plot of average knee temperature before and after activity

To analyse simple effects, a Student's t-test for paired samples with Bonferroni correction was used to test the differences between left and right leg during rest period and after exercise. Statistically significant difference was found in mean skin temperature $(p=0.043)$ after exercise, with left knee being cooler by $0.17( \pm 0.18){ }^{\circ} \mathrm{C}$ on average. There was no difference in temperature during the rest period before the exercise had started between the legs $(p=0,254)$.

Table 3. Difference in skin temperature between right and left knees

\begin{tabular}{|l|c|c|c|c|c|}
\hline & t & SE & df & $\boldsymbol{p}$ & corrected $\boldsymbol{p}$ \\
\hline Right vs left (rest) & 1.7 & 0.076 & 8 & 0.12 & 0.2544 \\
\hline Right vs left (after exercise) & 2.84 & 0.06 & 8 & 0.02 & 0.0434 \\
\hline
\end{tabular}

Interestingly, in this study we found a statistically significant, high negative correlation of BMI and resting knee temperatures Pearson's $r=-0.84$ and -0.78 , right (RLRest) and left leg (LLRest) respectively. The higher BMI value of the subject, the lower was average knee skin temperature. Correlogram can be seen in plot 2 .

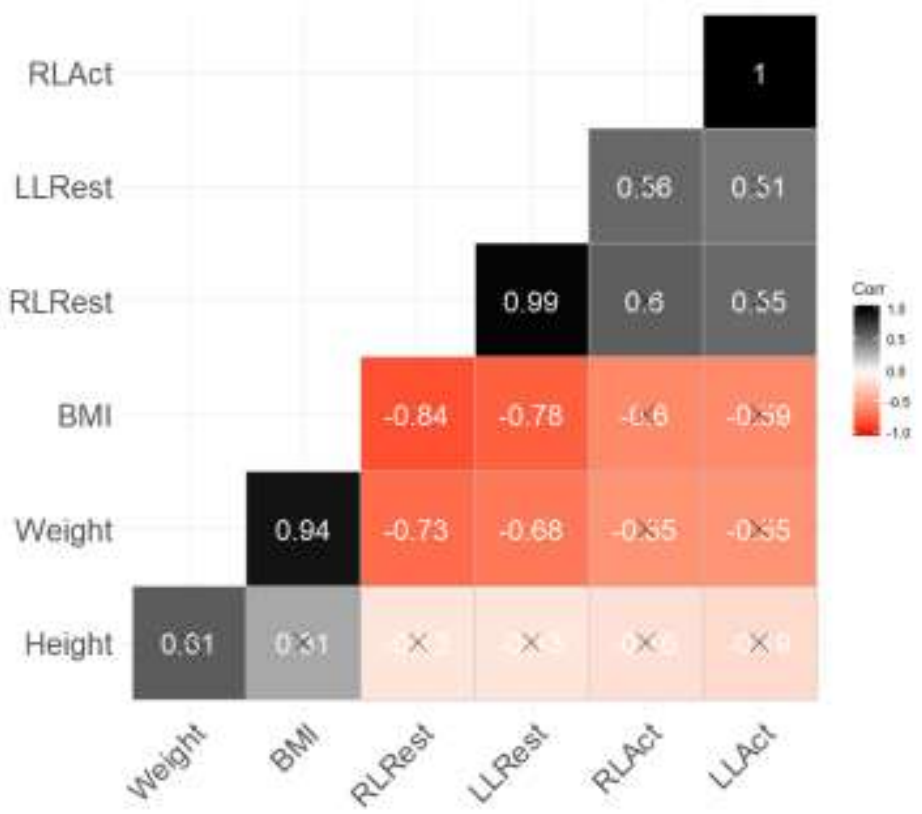

Plot 2. Correlogram of anthropometric and thermographic variables 
Most probable cause of this finding is the subcutaneous fat layer which acts like insulator keeping the skin cooler on the outside. This should be taken in to consideration in future similar studies.

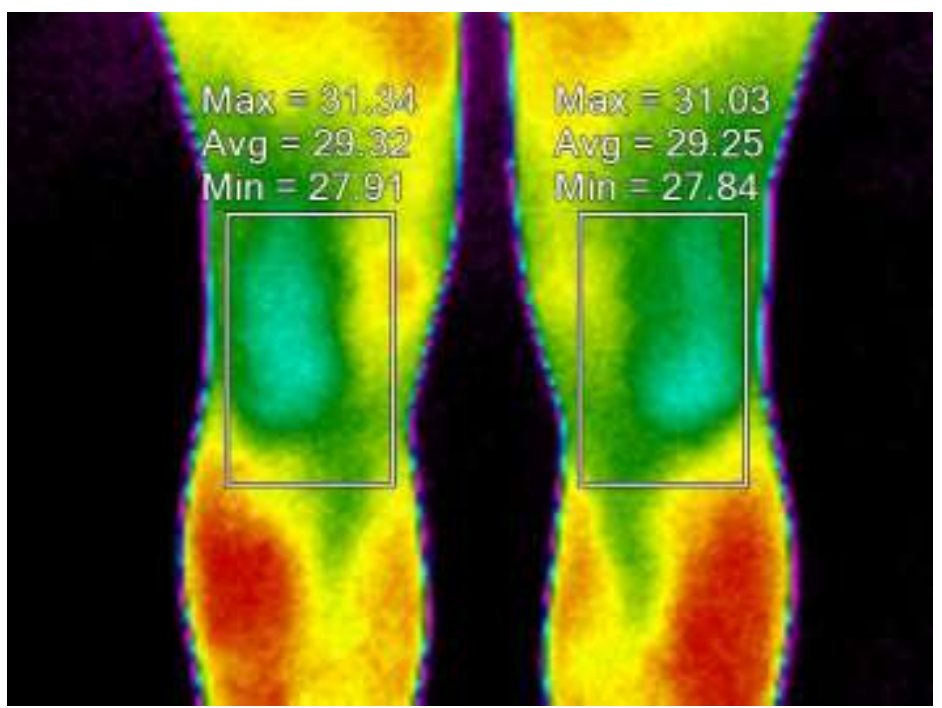

Fig. 1. Thermogram of both knees proposed by the Glamorgan protocol (anterior view); code BKA, measuring minimum, average and maximum temperatures.

\section{Conclusion}

This study proved the relation between physical activity and skin temperature with its influences on the initial fall in skin temperature over the working musculature and joints. This fall in skin temperature is associated with a higher demand of blood flow in the working musculature leading to the skin reflex vasoconstriction response of the blood vessels [10]. The higher demand for the blood flow results in more cooling of the skin at the beginning of the exercise. The longer the exercise, on the other hand, the weaker this effect gets while heat conduction begins to influence skin temperature. That is the reason why after a longer period of continuous exercise, skin starts to heat up. In addition, in this investigation, it was acknowledged that there was a small but significant difference in mean temperatures of left and right knee after physical exercise. Left knee was slightly cooler in seven out of total of nine subjects. This finding was also seen after the exercise on a stationary bicycle in the study of Švaic et al. [11]. The reason for these differences is probably due to the different usage of dominant and non-dominant leg during the running, although it is unclear whether the difference is mostly affected by heat production within the knee joint and working musculature or by skin blood vessel contractions. Unfortunately we did not have the information about the laterality of the subjects so we are unable to come to a conclusion about how the preference of one side of the body influences the temperature of left and right sides of the body during and after physical activity. Negative correlation between BMI and resting skin temperature which was acknowledged in this investigation give us a good idea on how the subcutaneous fat influences skin temperature. More precise correlations and models can be derived by direct measurement of the fat layer underneath the skin using anthropometric calipers. All these results give us a slightly new view of our standing point on how to analyse thermograms during physical activities since it is a dynamic process of skin temperature changes, musculature heat production and joint heat production, together with environmental conditions which all contribute both as regression and disturbing factors in sports and medicine thermography.

\section{REFERENCES}

[1] Merla A, Mattei PA, Di Donato L, Romani GL. Thermal imaging of cutaneous temperature modifications in runners during graded exercise. Ann Biomed Eng., vol. 38(1), p.p. 158-163, 2010.

[2] Čoh, M. i Širok, B., "Use of the thermovision method in sport training", Physical Education and Sport, vol. 5(1), p.p. 85-94, 2007.

[3] Merla, A., Mattei, P.A., Di Donato, L. \& Romani, G.L., "Thermal imaging of cutaneous temperature modifications in runners during graded exercise", Annals of biomedical engineering, vol. 38(1), p.p. 158-163, 2010.

[4] Chudecka, M. \& Lubkowska, A., "The use of thermal imaging to evaluate body temperature changes of athletes during training and a study on the impact of physiological and morphological factors on skin temperature", Human Movement, vol. 13(1), p.p. 33-39, 2012. 
[5] Vardasca, R. "Symmetry of temperature distribution in the upper and lower extremities", Thermology International, 18, 154-155, 2008.

[6] Hildebrandt, C., Raschner, C. \& Ammer, K. "An overview of recent application of medical infrared thermography in sports medicine in Austria", Sensors (Basel), 10(5), p.p. 4700-4715. Epub 2010.

[7] Adamczyk, J.G., Boguszewski, D. \& Siewierski, M. "Thermographic evaluation of lactate level in capillary blood during post-exercise recovery”, Kinesiology, 46(2), pp. 186-193, 2014.

[8] Seixas, A., Gonjo, T., Vardasca, R., Gabriel, J., Fernandes, R. \& Vilas-Boas, J.P. "A preliminary study on the relationship between energy expenditure and skin temperature in swimming", Proceedings of $12^{\text {th }}$ Quantitative Infrared Thermography Conference, paper QIRT2014-097, Bordeaux, France.

[9] Vučetić, V., Šentija, D., Sporiš, G., Trajković, N. \& Milanović, Z. "Comparison of ventilation threshold and heart rate defection point in fast and standard treadmill test protocols", Acta Clinica Croatica, 53, p.p. 190-203, 2014.

[10] Zontak, A., Sideman, S., Verbitsky, O. \& Beyar, R., "Dynamic thermography: analysis of hand temperature during exercise", Annals of Biomedical Engineering, vol. 26(6), p.p. 988-993, 1998.

[11] Švaić, V., Bolarić, A., Županić, D. \& Jurinjak, B. "Changes in skin temperature during interval type of physical exercise measured by infrared thermography", Proceedings of $13^{\text {th }}$ Quantitative Infrared Thermography Conference, paper QIRT2016-130, Gdansk, Poland. 